\title{
Invasive adenocarcinoma manifesting as pure ground glass nodule with different size: radiological characteristics differ while prognosis remains the same
}

\author{
Zijian Wang ${ }^{1 \#}$, Wei Zhu ${ }^{1 \#}$, Zhenzhen $\mathrm{Lu}^{2}$, Wei Li ${ }^{1}$, Jingyun Shi ${ }^{1}$ \\ ${ }^{1}$ Department of Radiology, Shanghai Pulmonary Hospital, Tongji University School of Medicine, Shanghai, China; ${ }^{2}$ Clinical Research Unit, \\ Shanghai Pulmonary Hospital, Tongji University School of Medicine, Shanghai, China \\ Contributions: (I) Conception and design: Z Wang, W Li, J Shi; (II) Administrative support: J Shi; (III) Provision of study materials or patients: Z \\ Wang, W Zhu; (IV) Collection and assembly of data: Z Wang, W Zhu; (V) Data analysis and interpretation: Z Wang, Z Lu, W Zhu; (VI) Manuscript \\ writing: All authors; (VII) Final approval of manuscript: All authors. \\ \#These authors contributed equally to this work. \\ Correspondence to: Wei Li; Jingyun Shi. Department of Radiology, Shanghai Pulmonary Hospital, Tongji University School of Medicine, 507 Zheng \\ Min Road, Shanghai 200433, China. Email: zgshznj@163.com; shijingyun89179@126.com.
}

\begin{abstract}
Background: Invasive adenocarcinoma (IA) manifesting as pure ground-glass nodule is rare and not been well studied. Meanwhile, tumor size is considered as a predictor of invasiveness in lung adenocarcinoma. The present study aimed to investigate the radiological and pathological characteristics as well as prognosis of IA manifesting as pure ground-glass nodule with different sizes.
\end{abstract}

Methods: Patients with solitary pure ground glass nodule (GGN) who underwent resection and were pathologically diagnosed as IA between July 2013 and July 2015 were included. Nodules were divided into four groups according to size: A, B, C, and D, corresponding to " $\leq 1 \mathrm{~cm}, "$ " $1-2 \mathrm{~cm}$, " " $2-3 \mathrm{~cm}$," and " $>3 \mathrm{~cm}$," respectively. The correlations and differences in radiological and pathological characteristics as well as prognosis among these groups were analyzed.

Results: The amounts of nodules in groups A, B, C, and D are 17, 148, 78, and 30, respectively. The average diameter of these 273 nodules is $1.9(1.5-2.4) \mathrm{cm}$. A large tumor is likely to have low computed tomography $(\mathrm{CT})$ value $(\mathrm{P}<0.001)$, irregular shape $(\mathrm{P}=0.001)$, spiculation appearance $(\mathrm{P}<0.001)$ and exhibit pleural indentation $(\mathrm{P}<0.001)$ and air bronchogram $(\mathrm{P}<0.001)$. The proportion of lepidic predominant adenocarcinoma (LPA) $(n=239,87.5 \%)$ is much higher than that of other subtypes $(n=34,12.5 \%)$. Currently, there is no case with lymphatic, pleural, or vessel invasion and lymph node involvement, and none died of recurrence or metastasis within 5 years after resection.

Conclusions: For IA manifesting as pure ground-glass nodule, size is correlated to invasiveness, and large tumors tend to have lower CT value, an irregular shape, lobulation and spiculation appearance and exhibit pleural indentation and air bronchogram. Nevertheless, the prognosis is excellent with $100 \% 5$-year diseasefree survival regardless of the size and pathological subtype.

Keywords: Invasive adenocarcinoma (IA); ground glass opacity (GGO); computed tomography (CT); prognosis

Submitted Jan 13, 2021. Accepted for publication May 06, 2021.

doi: $10.21037 /$ tcr-21-78

View this article at: https://dx.doi.org/10.21037/tcr-21-78

^ ORCID: 0000-0003-2702-7740. 


\section{Introduction}

Ground glass opacity (GGO) is defined as a hazy increased opacity of the lung with preservation of the bronchial and vascular margins (1). Persistently existing ground glass nodule (GGN) is reported as adenocarcinoma or precancerous lesion (2). According to the 2011 new classification of lung adenocarcinoma, pure GGN (pGGN), which is defined as focal nodular area composed purely of GGO and without internal solid components observed on thin section $(1 \mathrm{~mm})$ computed tomography (CT) images with lung window setting, could be pathologically diagnosed as atypical adenomatous hyperplasia (AAH), adenocarcinoma in situ (AIS), minimally invasive adenocarcinoma (MIA), and invasive adenocarcinoma (IA) (3). The probability of IA appearing as pGGN is $35.4-44.2 \%$, as reported previously (4-7).

Tumor size is one of the most important prognostic factors in clinical $\mathrm{T}$ descriptors (8), which is considered to be an independent predictor for survival $(9,10)$. The 8 th edition stage classification system for subsolid nodules indicate that part-solid nodules are staged according to the size of solid components, pGGNs less than $3.0 \mathrm{~cm}$ were classified as cTis, and pGGNs exceeding $3.0 \mathrm{~cm}$ were cT1a (11). Several studies have shown that the prognosis of lepidic predominant adenocarcinoma (LPA) is better than that of other pathological subtypes; the 5 -year disease free survival rate (DFS) can reach $>90 \%$ or even $100 \%(12,13)$. Due to the lack of solid component, which is associated with an invasive component, pGGN has a good prognosis; however, only a few studies have reported the prognosis of IA manifesting as pGGN with different sizes and pathological subtypes, as well as differences of radiological characteristics between this specific tumor with different size and pathological subtype.

In this retrospective study, a set of IA cases manifesting as pGGN were divided into groups according to tumor size, and we aimed to study the correlation and difference between the radiological and pathological characteristics as well as prognosis among these groups. We present the following article in accordance with the STROBE reporting checklist (available at https://dx.doi.org/10.21037/tcr-21-78).

\section{Methods}

The study was conducted in accordance with the Declaration of Helsinki (as revised in 2013). This retrospective study was approved by the Ethics Committee of Shanghai Pulmonary Hospital, Tongji University, China (K21-235). The requirement of informed consent was waived.

\section{Study population}

In the present retrospective study, we reviewed the medical records and CT data of patients with GGN who underwent surgical resection at Shanghai Pulmonary Hospital between July 2013 and July 2015. The inclusion criteria were existing GGNs and pathological diagnosis after resection. The exclusion criteria were as follows: (I) radiological evaluation of multiple nodules or mixed GGN (mGGN), which is defined as GGN with the presence of a solid component. (II) Pathologically diagnosed as AAH, AIS, MIA, benign lesions or malignancy other than adenocarcinoma. (III) Cases without 5 -year follow-up data are described below. The final cases were grouped according to the tumor size (group A: nodules with diameter $\leq 1 \mathrm{~cm}$; group B: nodules with diameter $1-2 \mathrm{~cm}$, but not include $1 \mathrm{~cm}$; group C: nodules with diameter $2-3 \mathrm{~cm}$, but not include $2 \mathrm{~cm}$; group D: nodules with diameter $>3 \mathrm{~cm}$ ). The medical records of each patient were reviewed for clinicopathological information as follows: age, sex, smoking status, pathology subtype, lymphatic invasion, vessel invasion, pleural invasion, lymph node involvement and radiological findings (as described below).

\section{Radiological evaluation}

The evaluation of radiological imaging was based on the last enhanced thin-section $(0.625-1 \mathrm{~mm})$ computed tomography (TSCT) scan before surgery. The CT data were independently reviewed on a Picture Archiving and Communication Systems (PACS) workstation with lung window images (window level, $-500 \mathrm{HU}$; window width, 1,500 $\mathrm{HU}$ ) and mediastinal window images (window level, $40 \mathrm{HU}$; window width, $400 \mathrm{HU}$ ). The presenting characteristics of pGGNs were determined by two radiologists (W. Li and J. Shi, with 8 and 29 years of diagnosis experience, respectively), blinded to the pathological and clinical data. When the evaluation of the two radiologists differed, the discrepancies were resolved by consensus.

The following parameters were evaluated on CT images: nodule location [right upper lobe (RUL), right middle lobe (RML), right lower lobe (RLL), left upper lobe (LUL), or left lower lobe (LLL)], nodule diameter (the longest 
diameter on axial CT images), mean CT attenuation (measured by placing three to five regions of interest (ROIs) within the pGGN while avoiding vessels and bronchioles), uniformity (homogeneous or heterogeneous), and morphological features, including shape (round/oval or irregular), margin (smooth or coarse), nodule-lung interface (well-defined or ill-defined), lobulation (defined as portion of nodule's surface shows wavy or scalloped configuration), spiculation (defined as the presence of strands extending from a nodule margin into the lung parenchyma without reaching the pleural surface), pleural indentation (defined as linear attenuation heading toward the pleura or the major or minor fissure from a nodule), and air bronchogram (defined as air-filled bronchi within a nodule).

\section{Pathological evaluation}

All clinical specimens were examined by experienced pathologists. For tumor sampling, the entire tumor tissue was obtained for each slide. Thus, each nodule was embedded for microscopic evaluation. The specimen of lung adenocarcinoma cases were reviewed according to the International Association for the Study of Lung Cancer/ American Thoracic Society/European Respiratory Society (IASLC/ATS/ERS) International Multidisciplinary Lung Adenocarcinoma Classification criteria (3), and the extent of existent tumor histological subtypes (AAH, AIS, MIA, and IA of lepidic, acinar, papillary, micropapillary, and solid patterns) was quantified to the nearest $5 \%$ level, adding up to a total of $100 \%$ subtype components per tumor. IA is present in at least one focus measuring more than $5 \mathrm{~mm}$ in greatest dimension. Invasion is defined as (I) histological subtypes other than a lepidic pattern (i.e., acinar, papillary, micropapillary, and/or solid) or (II) myofibroblastic stroma associated with invasive tumor cells. The diagnosis of LPA rather than MIA is made if the tumor (I) invades lymphatics, blood vessels, alveolar spaces or pleura or (II) contains tumor necrosis (III) spreads through alveolar spaces. Also, other characteristics, such as pleural invasion, lymphatic invasion, vascular invasion and lymph node involvement were recorded. According to the 8 th edition stage classification system for subsolid nodules, tumors showing pure lepidic growth without invasion are classified as pTis when total size measuring $3 \mathrm{~cm}$ or less and pT1a when total size measuring more than $3 \mathrm{~cm}$. tumors meet pathologic criteria for MIA are classified as pTimi when total size measuring $3 \mathrm{~cm}$ or less and $\mathrm{pT} 1 \mathrm{a}$ when total size measuring more than $3 \mathrm{~cm}$.

\section{Surgical treatment}

Lobectomy with systemic lymph node dissections was performed in 185 patients, segmentectomy with selective lymph node dissections was performed in 78 patients, and 10 patients underwent wedge resection with selective lymph node dissections. The resection margin was negative for the tumor in all patients.

\section{Follow-up}

All patients were scheduled for follow-up. Each patient was supposed to undergo a physical examination, chest CT scan, and blood tests every 6 months during the first 2 years postsurgery, followed by annual check-ups. Further evaluation was conducted by brain magnetic resonance imaging (MRI) and bone scintigraphy or positron emission tomography (PET) CT when any symptom or sign of recurrence or metastasis was observed. The follow-up period was from the date of surgery to July 1, 2020. The follow-up data were obtained by periodic reexamination and readmission $(n=243)$ in our hospital or telephonic interview $(n=30)$. The locoregional recurrence was defined as the evidence of tumor within the same lobe or the ipsilateral thoracic cavity and hilum or mediastinal lymph nodes. Distant recurrences were defined as the evidence of a tumor in another lobe, pleural space, or outside the hemithorax.

\section{Statistical analysis}

Clinical, radiological, and pathological evaluation data of nodules in each patient were collected. For continuous variables, the "mean \pm standard deviation $(\mathrm{SD})$ " was used for those that conformed to the normal distribution, and the median (quartile) was used for those with non-normal distribution. The categorical variables were reported as number and percentage. All the nodules were divided into four groups according to the diameter. The significant difference in CT attenuation was evaluated using one-way ANOVA, and differences in categorical variables such as sex, smoke history, nodule location, and shape were analyzed using Mantel-Haenszel chi-square test. The comparison between LPA and non-LPA groups in nodule size was analyzed using Mann-Whitney $U$ test, the difference in CT value was analyzed using Student's t-test, and differences in categorical variables were analyzed using chi-square test. No missing data were observed in the current study. $\mathrm{P}$ value $<0.05$ was considered statistically significant. All statistical 


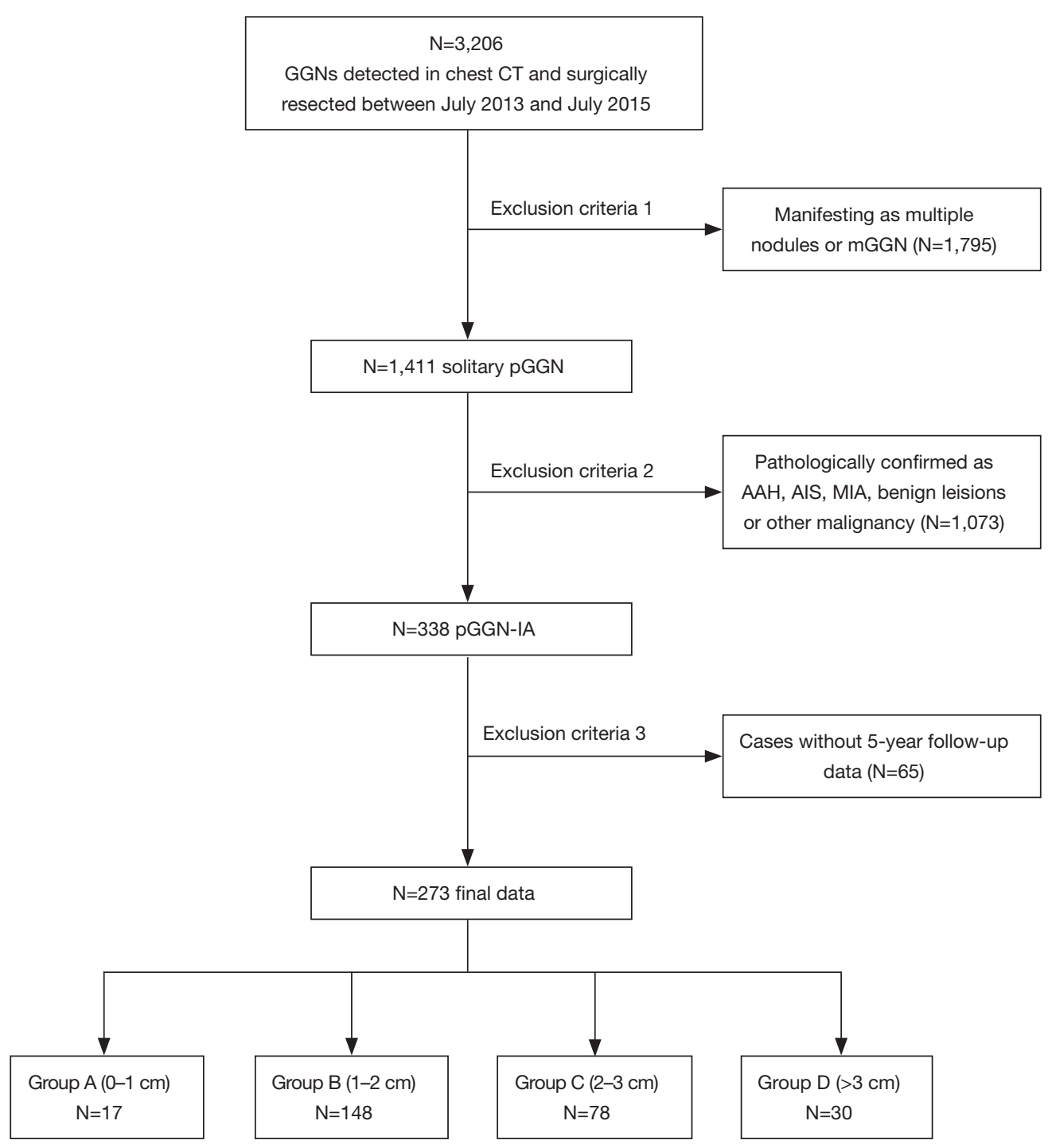

Figure 1 Flowchart shows case selection procedure. mGGN, mixed ground glass nodules; pGGN, pure ground glass nodule; AAH, atypical adenomatous hyperplasia; AIS, adenocarcinoma in situ; MIA, minimally invasive adenocarcinoma; IA, invasive adenocarcinoma.

analyses were performed using SPSS software (version 19.0, IBM Co., Armonk, NY, USA).

\section{Results}

\section{Clinical characteristics}

The population was evaluated, as shown in Figure 1. A total of 3,206 patients with GGNs, who underwent surgical resection at Shanghai Pulmonary Hospital between July 2013 and July 2015, were reviewed. Of these, 1,411 cases were identified as solitary pGGN and 1,795 cases included multiple nodules or mGGN. Among 1,411 solitary pGGN, 338 cases were pathologically diagnosed as IA and 1,073 cases of non-IA, and the final data included 273 cases after removal of 65 cases without 5 -year follow-up data. In this cohort, the proportion of IA in pGGN set was $24 \%(338 / 1,411)$. The number of nodules in groups $\mathrm{A}, \mathrm{B}, \mathrm{C}$, and $\mathrm{D}$ is $17,148,78$, and 30 , respectively. The clinical characteristics of these patients are summarized in Table 1; most of the patients were women ( $\mathrm{n}=170,62.3 \%)$, nonsmokers $(n=229,83.9 \%)$, and the average age of the cohort was 59 [52-64] years old. There is no one lost in the follow-up period, of all the patients, the median follow- 
Table 1 Clinical Characteristics of 273 invasive adenocarcinoma manifesting as pGGN

\begin{tabular}{|c|c|c|c|c|c|}
\hline Characteristics & Total $(n=273)$ & Group A $(n=17)$ & Group B $(n=148)$ & Group C $(n=78)$ & Group D $(n=30)$ \\
\hline \multicolumn{6}{|l|}{ Gender, n (\%) } \\
\hline Male & $103(37.7)$ & $1(5.9)$ & $51(34.5)$ & $38(48.7)$ & $13(43.3)$ \\
\hline Female & $170(62.3)$ & $16(94.1)$ & 97 (65.5) & $40(51.3)$ & $17(56.7)$ \\
\hline Yes & $44(16.1)$ & $1(5.9)$ & $24(16.2)$ & $15(19.2)$ & $4(13.3)$ \\
\hline No & 229 (83.9) & $16(94.1)$ & $124(83.8)$ & $63(80.8)$ & $26(86.7)$ \\
\hline
\end{tabular}

pGGN, pure ground glass nodule.

up was 68 [60-84] months, and none died of recurrence or metastasis within after resection.

\section{Radiological characteristics}

The radiological characteristics of the nodule are summarized in Table 2. The average diameter of these 273 nodules is $1.9(1.5-2.4) \mathrm{cm}$. Strikingly, cases with a diameter exceeding $1 \mathrm{~cm}$ comprise of high proportion, and more than half of all the cases were $1-2 \mathrm{~cm}(\mathrm{n}=148$, $54.2 \%)$. In addition, $30(11.0 \%)$ nodules had a diameter exceeding $3 \mathrm{~cm}$. The distribution of nodule size and boxplot of four groups are displayed in Figure 2. The majority of the nodules were localized in the upper lobe and often in the RUL ( $\mathrm{n}=106,38.8 \%)$. The mean CT value of all the nodules was $-511 \pm 104.5 \mathrm{HU}$, this parameter differed significantly among the four groups $(\mathrm{P}<0.001)$, and nodules with larger diameter tend to have a lower attenuation value.

In regard to morphological features, significant differences were detected in shape $(\mathrm{P}<0.001)$, lobulation $(\mathrm{P}=0.001)$, and spiculation $(\mathrm{P}<0.001)$, and large nodules were likely to have an irregular shape, lobulation, and spiculation appearance, while those with spiculation appearance were in a small proportion $(\mathrm{n}=20,7.3 \%)$. Figure 3 shows examples of pGGN in each group and some morphological features of these nodules. Pleural indentation and air bronchogram were observed in 148 (54.2\%) and 112 (41.0\%) cases, respectively, and these two signs are significantly frequently observed in large size groups $(\mathrm{P}<0.001)$. The examples of pleural indentation are shown in Figure 4. The majority of the nodules have heterogeneous density $(\mathrm{n}=172,63.0 \%)$, coarse margin $(n=163,59.7 \%)$ and a well-defined interface ( $\mathrm{n}=187,68.5 \%$ ), while no significant difference was detected among the four groups.

\section{Pathological characteristics}

The pathology subtype of the IA includes LPA, acinar predominant adenocarcinoma (APA), and papillary predominant adenocarcinoma (PPA). However, no case with lymphatic invasion, pleural invasion, vessel invasion and lymph node involvement were detected. The differences in the radiological and pathological characteristics between LPA and non-LPA are shown in Table 3; the proportion of LPA $(n=239,87.5 \%)$ is much higher than the other two subtypes $(\mathrm{n}=34,12.5 \%)$. Of all these radiological characteristics, only CT attenuation of nodules in the nonLPA group was significantly higher than those in the LPA group $(\mathrm{P}=0.011)$.

\section{Discussion}

To the best of our knowledge, this is the first largesample study regarding IA manifesting as pGGN. In the present study, the proportion of IA in pGGN sets is $24 \%$ $(338 / 1,411)$, which is lower than $35.4-44.2 \%$ reported in previous studies (4-7). Next, we further analyzed the distribution of nodule size and the radiological difference between various size groups and found that large tumors tend to have lower CT attenuation, irregular shape, lobulation, and spiculation appearance and exhibit pleural indentation and air bronchogram. In addition, most of the IA manifesting as pGGNs were pathologically diagnosed as LPA, and the prognosis was excellent with 100\% 5-year DFS regardless of the size and pathological subtype.

Tumor size is considered as a predictor of invasiveness in lung adenocarcinoma. Previous studies have focused on pGGN and found that the optimal cutoff value for differentiating preinvasive lesions and invasive lesions 
Table 2 Radiological and pathological characteristics of different groups

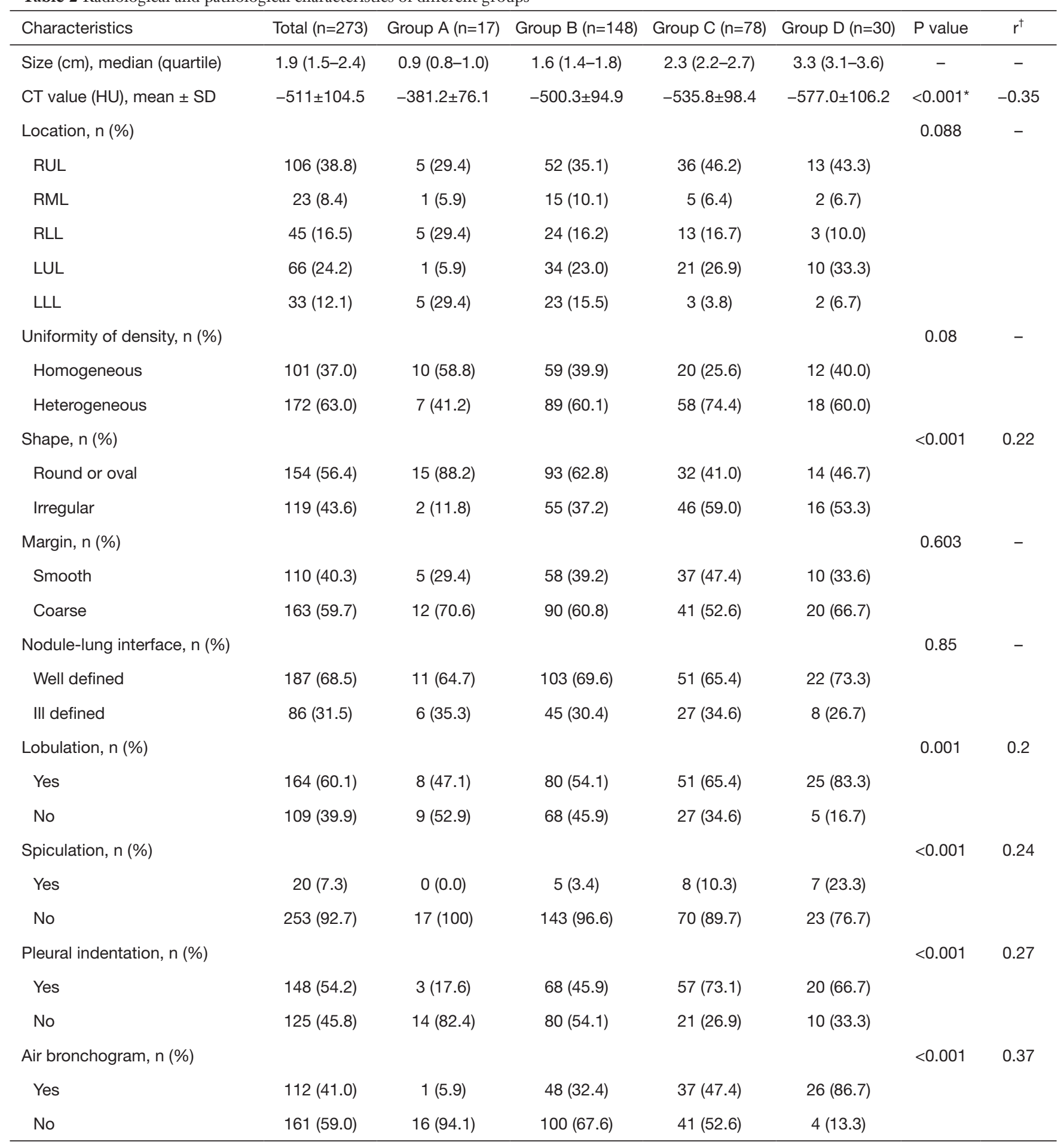

Table 2 (continued) 
Table 2 (continued)

\begin{tabular}{|c|c|c|c|c|c|c|c|}
\hline Characteristics & Total $(n=273)$ & Group A $(n=17)$ & Group B $(n=148)$ & Group C $(n=78)$ & Group D $(n=30)$ & $P$ value & $r^{\dagger}$ \\
\hline Lepidic & $239(87.5)$ & $13(76.5)$ & $132(89.2)$ & $69(88.5)$ & 25 (83.3) & & \\
\hline Acinar & $13(4.8)$ & $0(0.0)$ & $5(3.4)$ & $5(6.4)$ & $3(10.0)$ & & \\
\hline Papillary & $21(7.7)$ & $4(23.5)$ & $11(7.4)$ & $4(5.1)$ & $2(6.7)$ & & \\
\hline Solid & $0(0)$ & $0(0)$ & $0(0)$ & $0(0)$ & $0(0)$ & & \\
\hline Lymphatic invasion, n (\%) & $0(0)$ & $0(0)$ & $0(0)$ & $0(0)$ & $0(0)$ & - & - \\
\hline Pleural invasion, n (\%) & $0(0)$ & $0(0)$ & $0(0)$ & $0(0)$ & $0(0)$ & - & - \\
\hline Vessel invasion, n (\%) & $0(0)$ & $0(0)$ & $0(0)$ & $0(0)$ & $0(0)$ & - & - \\
\hline
\end{tabular}

$\mathrm{P}$ value in one-way ANOVA $\left(^{*}\right)$ or Mantel-Haenszel chi-square test. ${ }^{\dagger}, \mathrm{r}=$ coefficient of correlation. CT, computed tomography; DFS, disease free survival; SD, standard deviation; RUL, right upper lobe; RML, right middle lobe; RLL, right lower lobe; LUL, left upper lobe; LLL, left lower lobe.

A Size distribution of 273 pGGN

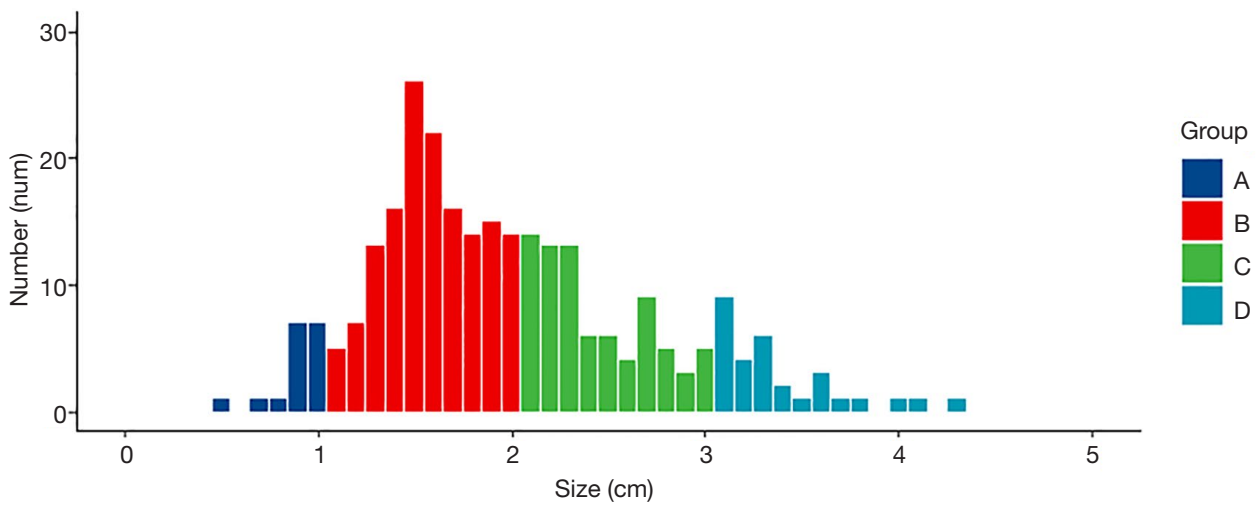

B Boxplot of four group
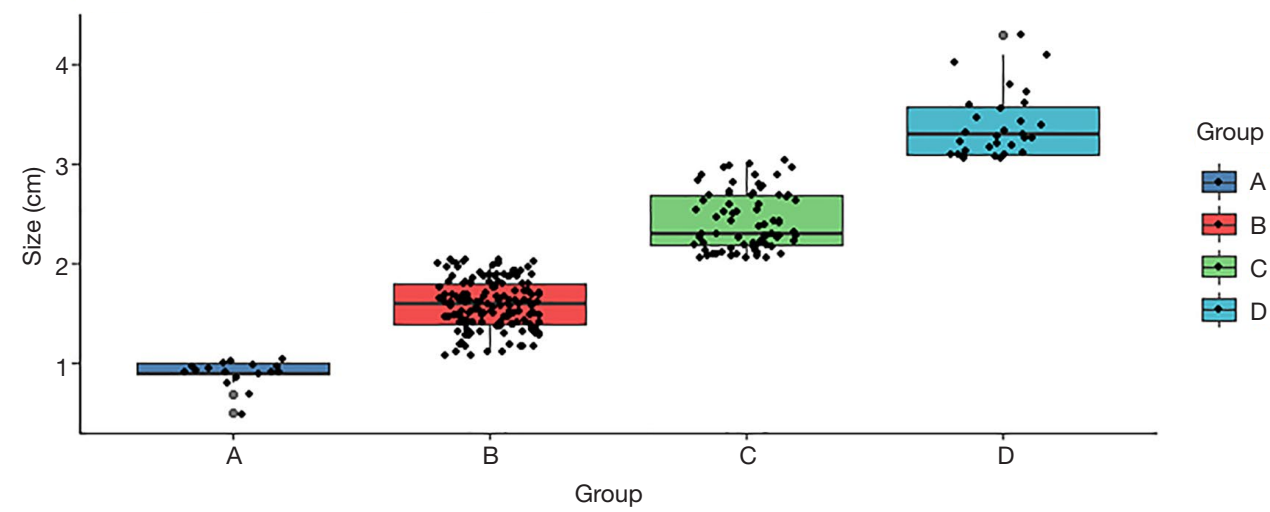

Figure 2 Figure (A) shows the size distribution of 273 pGGN and (B) shows the boxplot of four groups. pGGN, pure ground glass nodule. 


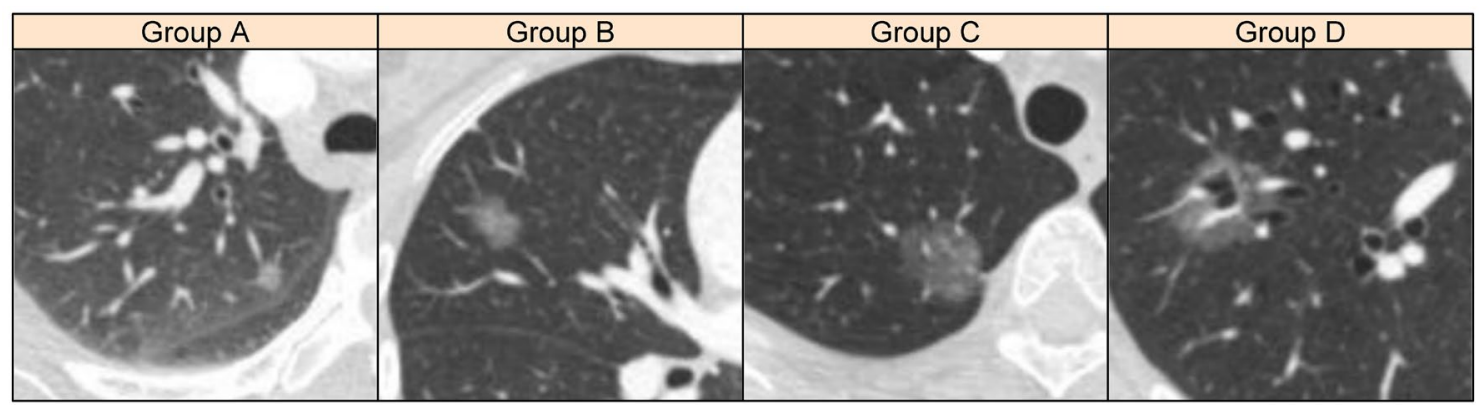

Figure 3 Examples of pGGN in each group. (A) pGGN in the right upper lobe in a 49-year-old woman with size of $0.8 \mathrm{~cm}$, oval shape and relatively higher CT attenuation. (B) pGGN in the right middle lobe in a 56-year-old woman with size of $1.5 \mathrm{~cm}$, well-defined interface and lobulation appearance. (C) pGGN in the right upper lobe in a 62-year-old woman with diameter of $2.3 \mathrm{~cm}$, smooth margin and homogenous density. (D) pGGN in the right lower lobe in a 61-year-old man with diameter of $3.1 \mathrm{~cm}$ and heterogenous density. pGGN, pure ground glass nodule.

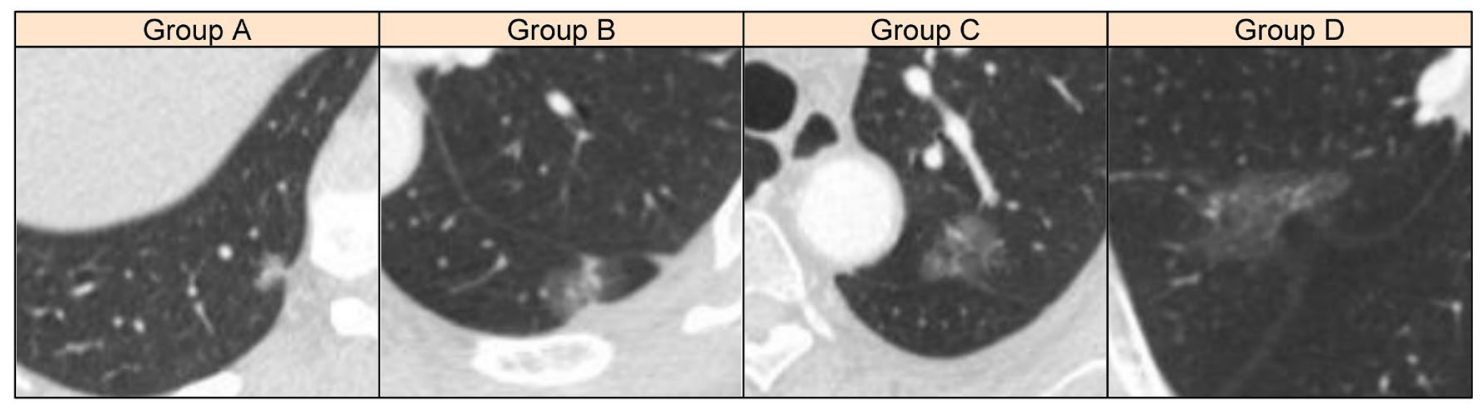

Figure 4 Examples of pGGN with pleural indentation in each group. Nodules with different size are close to the pleura and show pleural indentation on CT, but there is no case with pleural invasion. pGGN, pure ground glass nodule.

was around $10 \mathrm{~mm}(6,14,15)$. The current study showed a similar result since most IA cases had tumor size $>1 \mathrm{~cm}$. Also, IA manifesting as pGGN with a large size is rare because of the high possibility of the presence of a solid component in GGN. In the study by Li et al. (16), only 22 pGGNs were detected among 304 IAs manifesting GGO with size $>3 \mathrm{~cm}$.

The CT value is considered as another predictor of invasiveness. Previous studies have reported that the mean CT attenuation of pGGN in the IA group is -512 or -507 $\mathrm{HU}(5,6)$. In the present study, the mean CT value of these nodules is $-511 \mathrm{HU}$, which is consistent with that reported previously. Notably, the mean CT value of group $\mathrm{A}$ is significantly higher than that in the other groups. A high CT attenuation of pGGN indicates the proliferation of a large number of tumor cells in the interstitial tissue (17). Based on the pathology of IA, smaller nodules may exhibit higher CT value than bigger nodules due to the close arrangement of tumor cells. However, small pGGNs were usually not suitable for resection, unless these nodules reveal increased density or other characteristics and cause panic in the patients, this could result in higher CT value in resected cases. Furthermore, a high CT value of nonLPA may reflect more invasive component and tumor cell proliferation in the interstitial tissue. Therefore, we have to precaution $\mathrm{pGGN}$ with size $<1 \mathrm{~cm}$ and relatively high CT attenuation, which has certain possibility for being IA.

In the present study, a significant difference was detected in other characteristics, such as shape, lobulation, spiculation, pleural indentation, and air bronchogram. Several studies reported that these characteristics were associated with invasive lesions $(15,18-22)$, and the current study revealed that these features are frequently observed in large-size groups. Previous studies reveal that the nodule size is associated with the invasiveness of lung adenocarcinoma $(6,23)$, the current study shows that this 
Table 3 CT and pathological characteristics of LPA group and non-LPA group

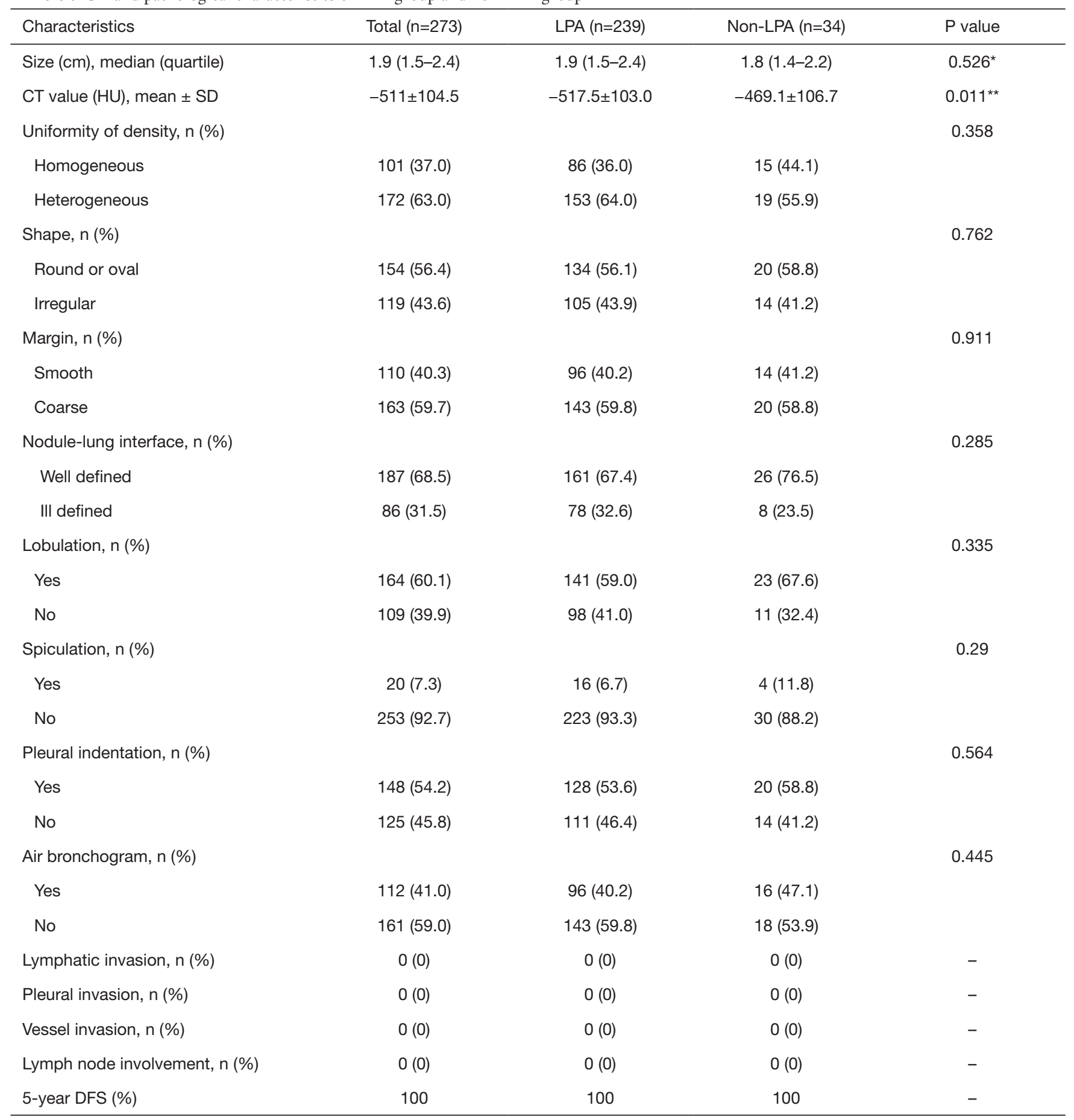

$\mathrm{P}$ value in Mann-Whitney $\mathrm{U}$ test $\left(^{*}\right)$, Student's $t$-test $\left(^{\star \star}\right)$ or chi-square test. LPA, lepidic predominant adenocarcinoma; CT, computed tomography; SD, standard deviation; DFS, disease free survival. 
conclusion also applies to IA manifesting as pGGN: larger size nodules are more aggressive and likely to exhibit CT characteristics that are frequently seen in invasive cases. Moreover, the present study indicated that pleural invasion is rare even in the presence of pleural indentation on CT, which was consistent with that found previously (21).

The GGO component on CT is associated with lepidic growth pattern, which is noninvasive in many cases, and tumors manifesting as pGGN have a good prognosis. In the study by Mao et al. (24), the 5-year overall survival (OS) and DFS rates of the pGGN group were $100 \%$ and $99.1 \%$, respectively. In the present study, no one died of recurrence or metastasis within 5 years after resection, even though the nodules were pathological IA, the prognosis of this cohort was satisfactory with a 5 -year DFS rate reaching $100 \%$. The pathological subtype of IA was a predictor of prognosis, among which LPA showed maximal 5 -year DFS of $>90 \%$ and even $100 \%(12,13)$. In the present study, $239(87.5 \%)$ cases are pathologically diagnosed as LPA, and there is no case of micropapillary predominant adenocarcinoma (MPA) or solid predominant adenocarcinoma (SPA), which correspond to poor prognosis. Moreover, patients with tumors that were pathologically diagnosed as nonLPA also showed a good prognosis regardless of the tumor size. Some other aggressive tumor behaviors like lymphatic invasion, pleural invasion, vessel invasion and lymph node involvement are not observed in all these 273 cases. As discussed above, the pathological characteristics, including lepidic predominant growth pattern and absence of lymphatic, pleural, vessel invasion and lymph node involvement, contribute to a favorable prognosis in patients with IA manifested as pGGN.

According to the 8th edition of the TNM classification of non-small cell lung carcinoma (NSCLC) (11), tumor size is one of the main elements of T staging, which is significant in the prognosis of lung adenocarcinoma. The clinical $\mathrm{T}$ stage was dependent on the solid component size excluding the GGO component rather than the total size $(11,25)$. Moreover, in lung adenocarcinomas with a subsolid appearance, many studies have demonstrated that the invasive size rather than total size is a better predictor for survival (11,26-29). In the present study, these nodules were identified as cTis and cT1a stage, which were considered to be stage 0 and stage I A1 tumors. According to the study in the IASLC lung cancer staging project, the prognosis of stage I A1 lung cancer was significantly better than the other higher stages with a 5 -year OS of $92 \%$ (30), while the prognosis in the current study was improved. This could be ascribed to the reason that the stage I A1 tumor in the former study included solid nodule $<1 \mathrm{~cm}$ or subsolid nodules with solid components $<1 \mathrm{~cm}$. The results in the present study indicated that IA manifesting as pGGNs have a good prognosis regardless of tumor size and indirectly demonstrated the superiority of the 8th edition of the TNM classification of NSCLC. However, whether pGGN should be removed or not, and what surgical treatment should be adopted, is still need to be confirmed by the results of more prospective studies.

Nevertheless, the present study has several limitations. The first limitation is that data are derived from a single institution and a small number of patients, especially in groups A and D, while selection bias could be attributed to the retrospective study design. The second limitation is that the present study lacks evaluation of the quantitative parameters, such as volume and mass, which are frequently used in the former studies $(4,31)$. In addition, the evaluation of some CT features, such as homogeneity and spiculation might be subjective and ambiguous, inevitably leading to bias.

In conclusion, for IA manifesting as pGGN, size is correlated to invasiveness, large tumors tend to have lower CT value, an irregular shape, lobulation and spiculation appearance and exhibit pleural indentation and air bronchogram. Nevertheless, the prognosis is excellent with $100 \%$ 5-year DFS irrespective of the size and pathological subtype.

\section{Acknowledgments}

We thank Mr. Saroj of MedSci for helping us with polishing the manuscript.

Funding: This work was supported by Clinical Research Foundation of Shanghai Pulmonary Hospital (FK1944).

\section{Footnote}

Reporting Checklist: The authors have completed the STROBE reporting checklist. Available at https://dx.doi. org/10.21037/tcr-21-78

Data Sharing Statement: Available at https://dx.doi. org/10.21037/tcr-21-78

Peer Review File: Available at https://dx.doi.org/10.21037/ tcr-21-78

Conflicts of Interest: All authors have completed the ICMJE 
uniform disclosure form (available at https://dx.doi. org/10.21037/tcr-21-78). The authors report funding from Shanghai Pulmonary Hospital.

Ethical Statement: The authors are accountable for all aspects of the work in ensuring that questions related to the accuracy or integrity of any part of the work are appropriately investigated and resolved. The study was conducted in accordance with the Declaration of Helsinki (as revised in 2013). This retrospective study was approved by the Ethics Committee of Shanghai Pulmonary Hospital, Tongji University, China (K21-235). The requirement of informed consent was waived.

Open Access Statement: This is an Open Access article distributed in accordance with the Creative Commons Attribution-NonCommercial-NoDerivs 4.0 International License (CC BY-NC-ND 4.0), which permits the noncommercial replication and distribution of the article with the strict proviso that no changes or edits are made and the original work is properly cited (including links to both the formal publication through the relevant DOI and the license). See: https://creativecommons.org/licenses/by-nc-nd/4.0/.

\section{References}

1. Godoy MC, Naidich DP. Subsolid pulmonary nodules and the spectrum of peripheral adenocarcinomas of the lung: recommended interim guidelines for assessment and management. Radiology 2009;253:606-22.

2. Son JY, Lee HY, Kim JH, et al. Quantitative CT analysis of pulmonary ground-glass opacity nodules for distinguishing invasive adenocarcinoma from non-invasive or minimally invasive adenocarcinoma: the added value of using iodine mapping. Eur Radiol 2016;26:43-54.

3. Travis WD, Brambilla E, Noguchi M, et al. International Association for the Study of Lung Cancer/American Thoracic Society/European Respiratory Society: international multidisciplinary classification of lung adenocarcinoma: executive summary. Proc Am Thorac Soc 2011;8:381-5.

4. Liu Y, Sun H, Zhou F, et al. Imaging features of TSCT predict the classification of pulmonary preinvasive lesion, minimally and invasive adenocarcinoma presented as ground glass nodules. Lung Cancer 2017;108:192-7.

5. Lim HJ, Ahn S, Lee KS, et al. Persistent pure ground-glass opacity lung nodules $\geq 10 \mathrm{~mm}$ in diameter at CT scan: histopathologic comparisons and prognostic implications.
Chest 2013;144:1291-9.

6. Jin X, Zhao SH, Gao J, et al. CT characteristics and pathological implications of early stage (T1NOM0) lung adenocarcinoma with pure ground-glass opacity. Eur Radiol 2015;25:2532-40.

7. She $\mathrm{Y}$, Zhao L, Dai C, et al. Preoperative nomogram for identifying invasive pulmonary adenocarcinoma in patients with pure ground-glass nodule: A multi-institutional study. Oncotarget 2017;8:17229-38.

8. Goldstraw P, Crowley J, Chansky K, et al. The IASLC Lung Cancer Staging Project: proposals for the revision of the TNM stage groupings in the forthcoming (seventh) edition of the TNM Classification of malignant tumours. J Thorac Oncol 2007;2:706-14.

9. Zhang J, Gold KA, Lin HY, et al. Relationship between tumor size and survival in non-small-cell lung cancer (NSCLC): an analysis of the surveillance, epidemiology, and end results (SEER) registry. J Thorac Oncol 2015;10:682-90.

10. Morgensztern D, Waqar S, Subramanian J, et al. Prognostic significance of tumor size in patients with stage III non-small-cell lung cancer: a surveillance, epidemiology, and end results (SEER) survey from 1998 to 2003. J Thorac Oncol 2012;7:1479-84.

11. Travis WD, Asamura H, Bankier AA, et al. The IASLC Lung Cancer Staging Project: Proposals for Coding T Categories for Subsolid Nodules and Assessment of Tumor Size in Part-Solid Tumors in the Forthcoming Eighth Edition of the TNM Classification of Lung Cancer. J Thorac Oncol 2016;11:1204-23.

12. Russell PA, Wainer Z, Wright GM, et al. Does lung adenocarcinoma subtype predict patient survival?: A clinicopathologic study based on the new International Association for the Study of Lung Cancer/American Thoracic Society/European Respiratory Society international multidisciplinary lung adenocarcinoma classification. J Thorac Oncol 2011;6:1496-504.

13. Xu CH, Wang W, Wei $Y$, et al. Prognostic value of the new International Association for the Study of Lung Cancer/American Thoracic Society/European Respiratory Society classification in stage IB lung adenocarcinoma. Eur J Surg Oncol 2015;41:1430-6.

14. Lee SM, Park CM, Goo JM, et al. Invasive pulmonary adenocarcinomas versus preinvasive lesions appearing as ground-glass nodules: differentiation by using CT features. Radiology 2013;268:265-73.

15. Chu ZG, Li WJ, Fu BJ, et al. CT Characteristics for Predicting Invasiveness in Pulmonary Pure Ground-Glass 
Nodules. AJR Am J Roentgenol 2020;215:351-8.

16. Li W, Zhou F, Wan Z, et al. Clinicopathologic features and lymph node metastatic characteristics in patients with adenocarcinoma manifesting as part-solid nodule exceeding $3 \mathrm{~cm}$ in diameter. Lung Cancer 2019;136:37-44.

17. Noguchi M. Stepwise progression of pulmonary adenocarcinoma--clinical and molecular implications. Cancer Metastasis Rev 2010;29:15-21.

18. Ye T, Deng L, Wang S, et al. Lung Adenocarcinomas Manifesting as Radiological Part-Solid Nodules Define a Special Clinical Subtype. J Thorac Oncol 2019;14:617-27.

19. Yanagawa M, Tsubamoto M, Satoh Y, et al. Lung Adenocarcinoma at CT with $0.25-\mathrm{mm}$ Section Thickness and a 2048 Matrix: High-Spatial-Resolution Imaging for Predicting Invasiveness. Radiology 2020;297:462-71.

20. Zhang T, Pu XH, Yuan M, et al. Histogram analysis combined with morphological characteristics to discriminate adenocarcinoma in situ or minimally invasive adenocarcinoma from invasive adenocarcinoma appearing as pure ground-glass nodule. Eur J Radiol 2019;113:238-44.

21. Zhao Q, Wang JW, Yang L, et al. CT diagnosis of pleural and stromal invasion in malignant subpleural pure ground-glass nodules: an exploratory study. Eur Radiol 2019;29:279-86.

22. Qiu T, Ru X, Yin K, et al. Two nomograms based on CT features to predict tumor invasiveness of pulmonary adenocarcinoma and growth in pure GGN: a retrospective analysis. Jpn J Radiol 2020;38:761-70.

23. Lee HY, Choi YL, Lee KS, et al. Pure ground-glass opacity neoplastic lung nodules: histopathology, imaging, and management. AJR Am J Roentgenol 2014;202:W224-33.

24. Mao R, She Y, Zhu E, et al. A Proposal for Restaging of Invasive Lung Adenocarcinoma Manifesting as Pure Ground Glass Opacity. Ann Thorac Surg

Cite this article as: Wang Z, Zhu W, Lu Z, Li W, Shi $\mathrm{J}$. Invasive adenocarcinoma manifesting as pure ground glass nodule with different size: radiological characteristics differ while prognosis remains the same. Transl Cancer Res 2021;10(6):2755-2766. doi: 10.21037/tcr-21-78
2019;107:1523-31.

25. Rami-Porta R, Bolejack V, Crowley J, et al. The IASLC Lung Cancer Staging Project: Proposals for the Revisions of the T Descriptors in the Forthcoming Eighth Edition of the TNM Classification for Lung Cancer. J Thorac Oncol 2015;10:990-1003.

26. Rami-Porta R, Ball D, Crowley J, et al. The IASLC Lung Cancer Staging Project: proposals for the revision of the $\mathrm{T}$ descriptors in the forthcoming (seventh) edition of the TNM classification for lung cancer. J Thorac Oncol 2007;2:593-602.

27. Kadota K, Villena-Vargas J, Yoshizawa A, et al. Prognostic significance of adenocarcinoma in situ, minimally invasive adenocarcinoma, and nonmucinous lepidic predominant invasive adenocarcinoma of the lung in patients with stage I disease. Am J Surg Pathol 2014;38:448-60.

28. Yanagawa N, Shiono S, Abiko M, et al. The correlation of the International Association for the Study of Lung Cancer (IASLC)/American Thoracic Society (ATS)/European Respiratory Society (ERS) classification with prognosis and EGFR mutation in lung adenocarcinoma. Ann Thorac Surg 2014;98:453-8.

29. Maeyashiki T, Suzuki K, Hattori A, et al. The size of consolidation on thin-section computed tomography is a better predictor of survival than the maximum tumour dimension in resectable lung cancer. Eur J Cardiothorac Surg 2013;43:915-8.

30. Chansky K, Detterbeck FC, Nicholson AG, et al. The IASLC Lung Cancer Staging Project: External Validation of the Revision of the TNM Stage Groupings in the Eighth Edition of the TNM Classification of Lung Cancer. J Thorac Oncol 2017;12:1109-21.

31. Qi LL, Wu BT, Tang W, et al. Long-term follow-up of persistent pulmonary pure ground-glass nodules with deep learning-assisted nodule segmentation. Eur Radiol 2020;30:744-55. 